\title{
An intelligent shopping list based on the application of partitioning and machine learning algorithms
}

Nadia Tahiri ${ }^{1}$, Bogdan Mazoure ${ }^{2}$ and Vladimir Makarenkov ${ }^{1}$

Université du Québec à Montréal and ${ }^{2}$ McGill University

Objectives
- Predict which groceries the consumer will want
to buy again or will try to buy for the first
time, and in which store(s) in a given area he
will shop;
- Create a smart shopping list by offering the
consumer a personalized weekly shopping list
based on their shopping history and current
promotions;
- Depending on the user $u$ and the user's
purchase history (order
the probability that a product $i$ is included in
the next order $r_{t+1}$ of $u$.

\section{Introduction}

A shopping list is an integral part of the shopping experience for many consumers. A typical grocery retailer offers consumers thousands of promotions every week to attract more consumers and thus improve their sales [1].

\section{Dataset}

Figure 1: Website CircuitPromo.ca with the postal code H2Y $1 \mathrm{C} 6$ in Montreal
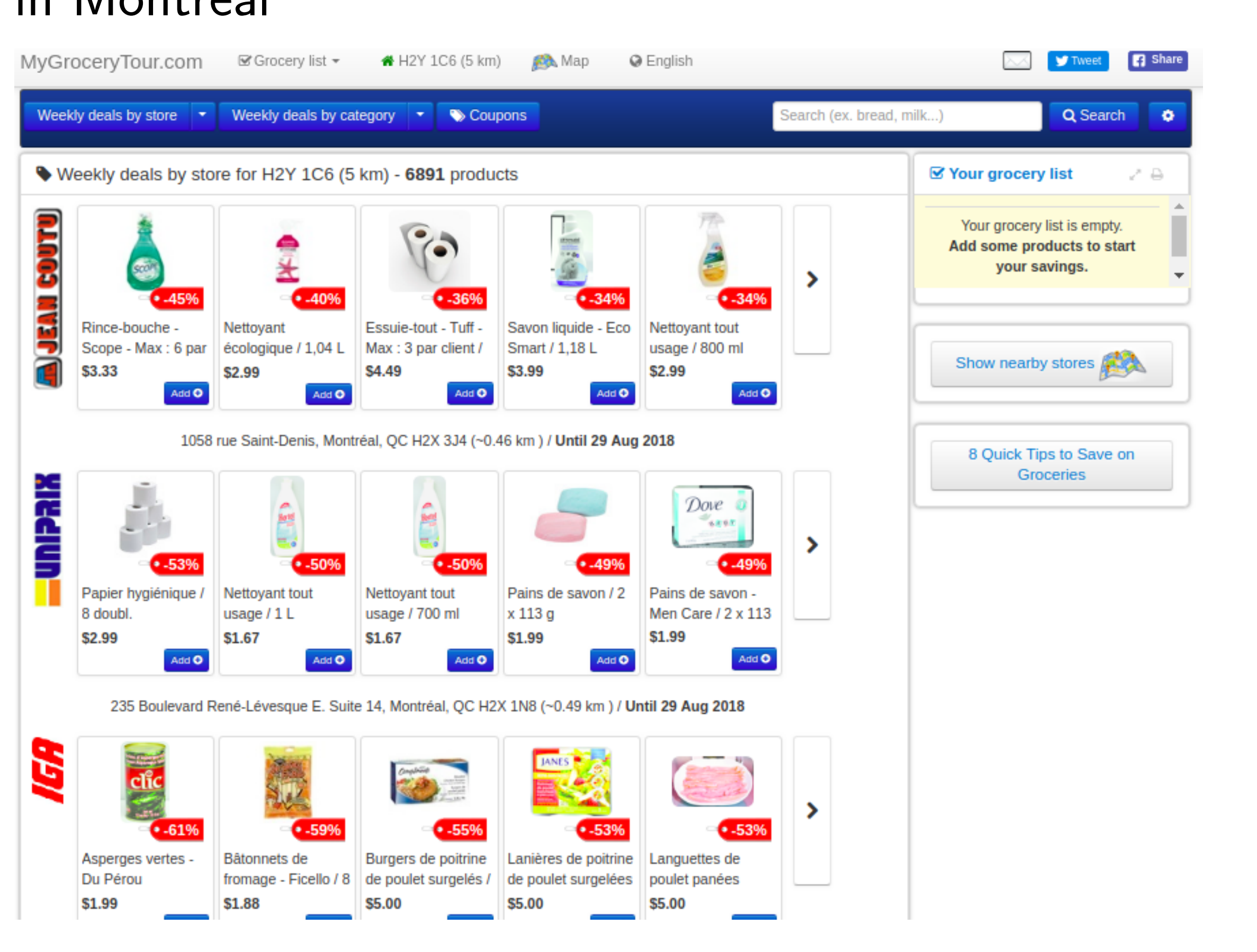

Methods

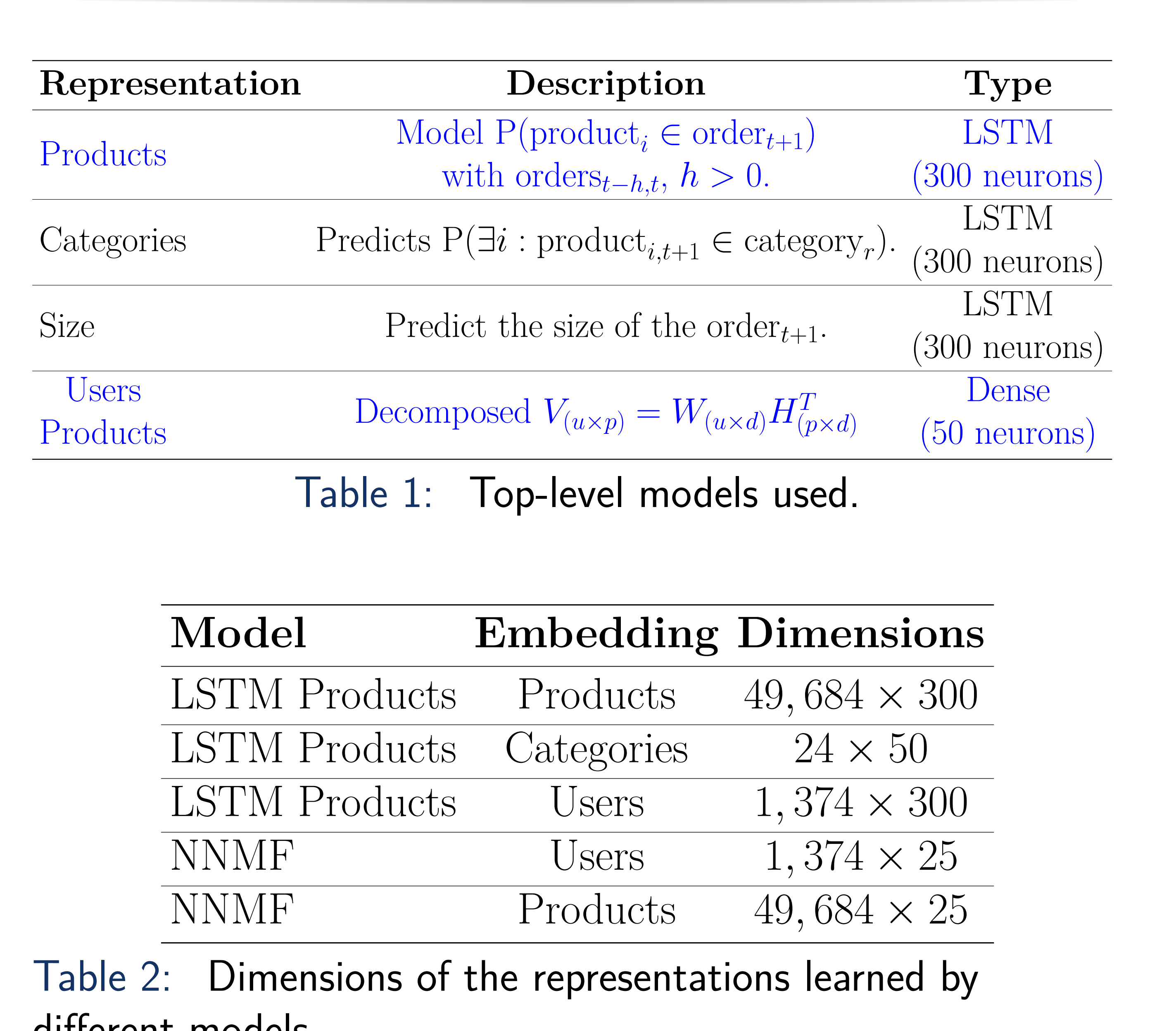

Table 2: Dimensions of the representations learned by

Products/Baskets

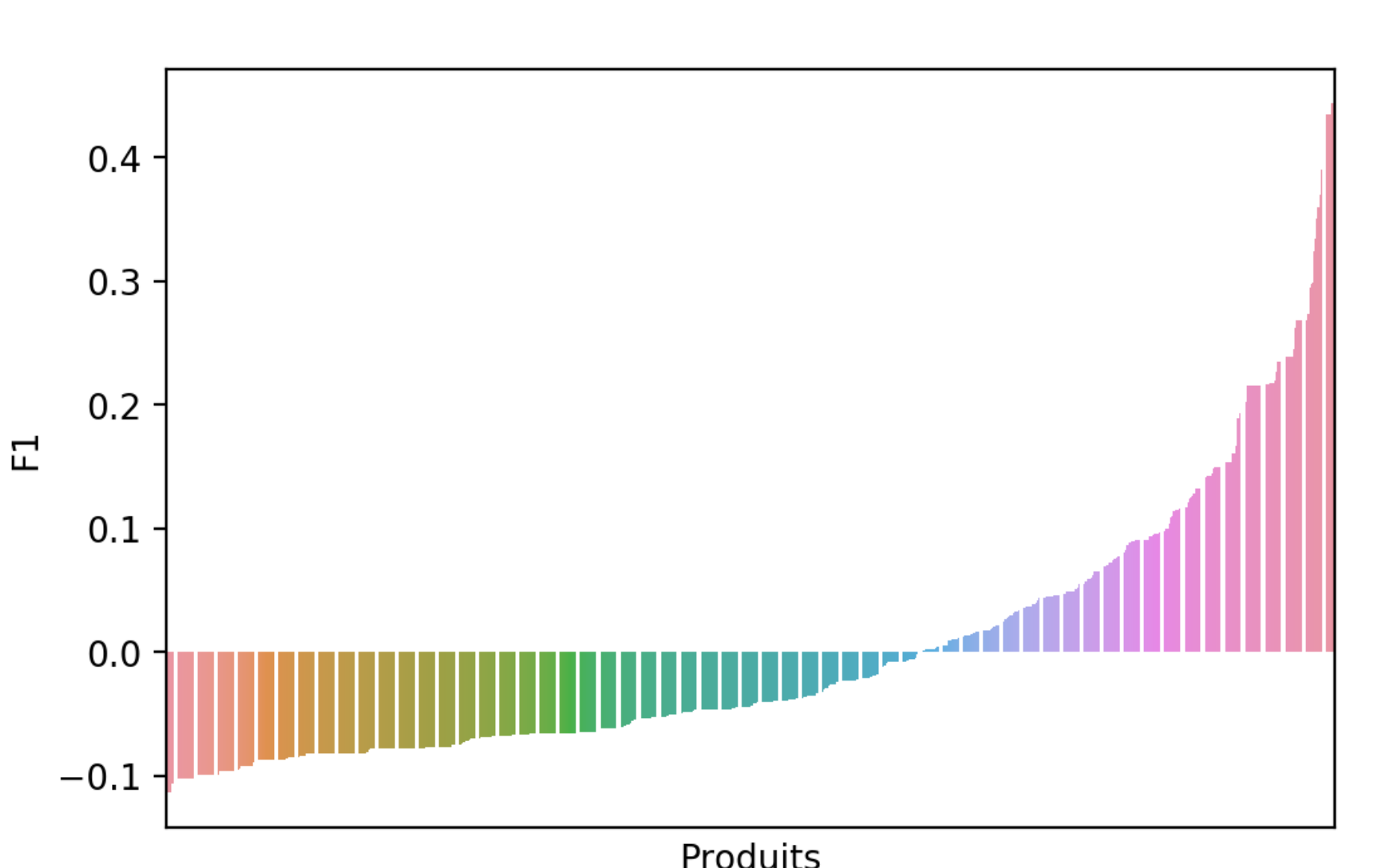

Figure 2: Distribution of $F_{1}$ measures relative to products, around average.

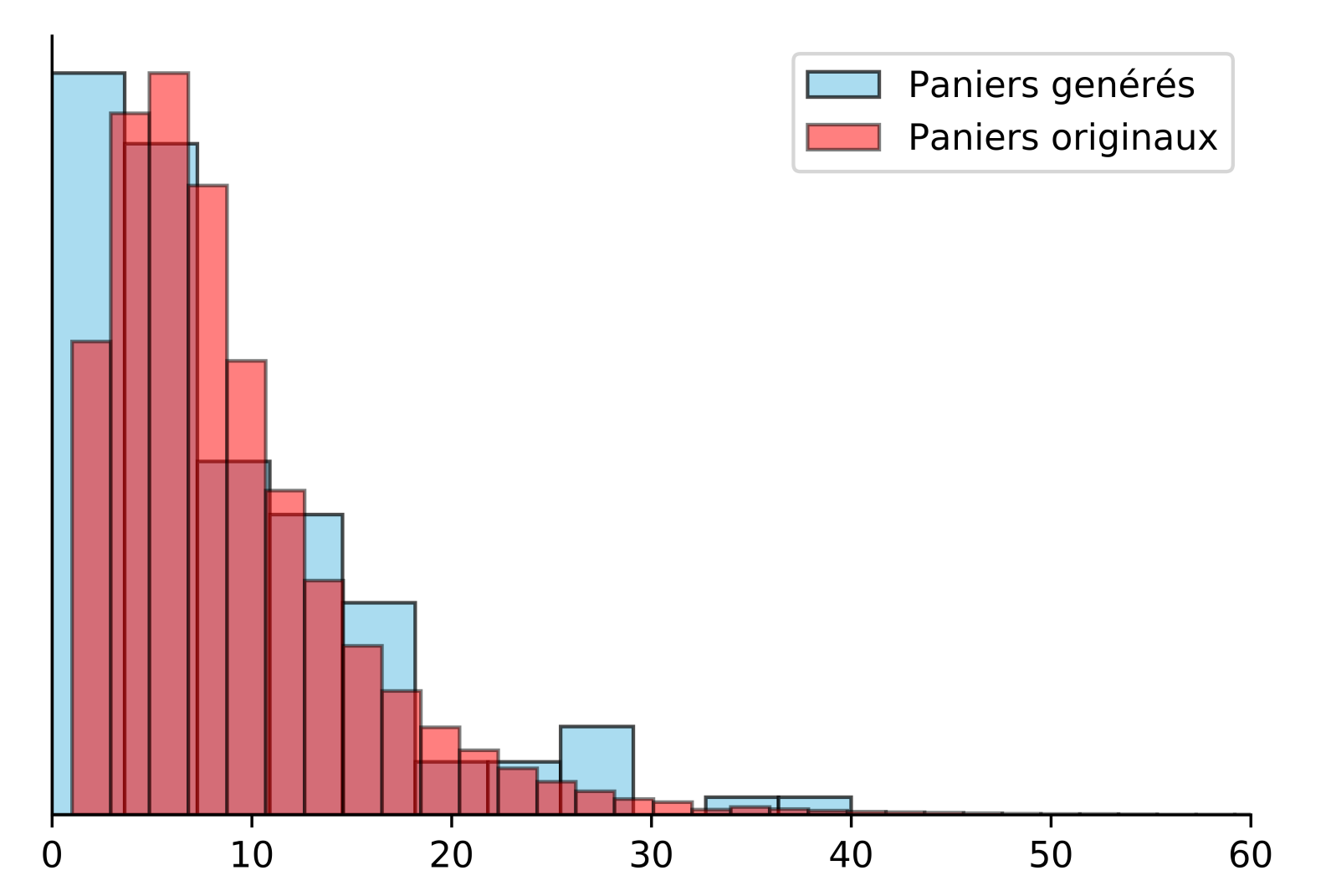

Figure 3: Distribution of the size of the predicted and original baskets.
Validation step

The final basket is chosen according to the final probabilities of reorganization, choosing the subset of products with the maximum expected $F_{1}$ score $[2]$.

$\max _{\mathcal{P}} \mathbb{E}_{p^{\prime} \in \mathcal{P}}\left[F_{1}(\mathcal{P})\right]=\max _{\mathcal{P}} \mathbb{E}_{p^{\prime} \in \mathcal{P}}\left[\frac{2 \Sigma_{i \in \mathcal{P}} \operatorname{TP}(i)}{\Sigma_{i \in \mathcal{P}}(2 \mathrm{VP}(i)+\mathrm{FN}(i)+\mathrm{FP}(i))}\right.$,

where $\mathrm{TP}=\mathbb{I}[\lfloor p(i)\rceil=1] \mathbb{I}\left[R_{i}=1\right]$,

$\mathrm{FN}=\mathbb{I}[\lfloor p(i)]=0] \mathbb{I}\left[R_{i}=1\right]$

$\mathrm{FP}=\mathbb{I}[\mid p(i)]=1] \mathbb{[}\left[R_{i}=0\right]$ and

$R_{i}=1$ if the product $i$ was bought in the cart $p^{\prime} \in \mathcal{P}$, otherwise 0 .

We was used $\mathbb{E}_{X}\left[F_{1}(Y)\right]=\underset{x \in X}{\Sigma} F_{1}(Y=y \mid x) P(X=$

\section{Consumers}

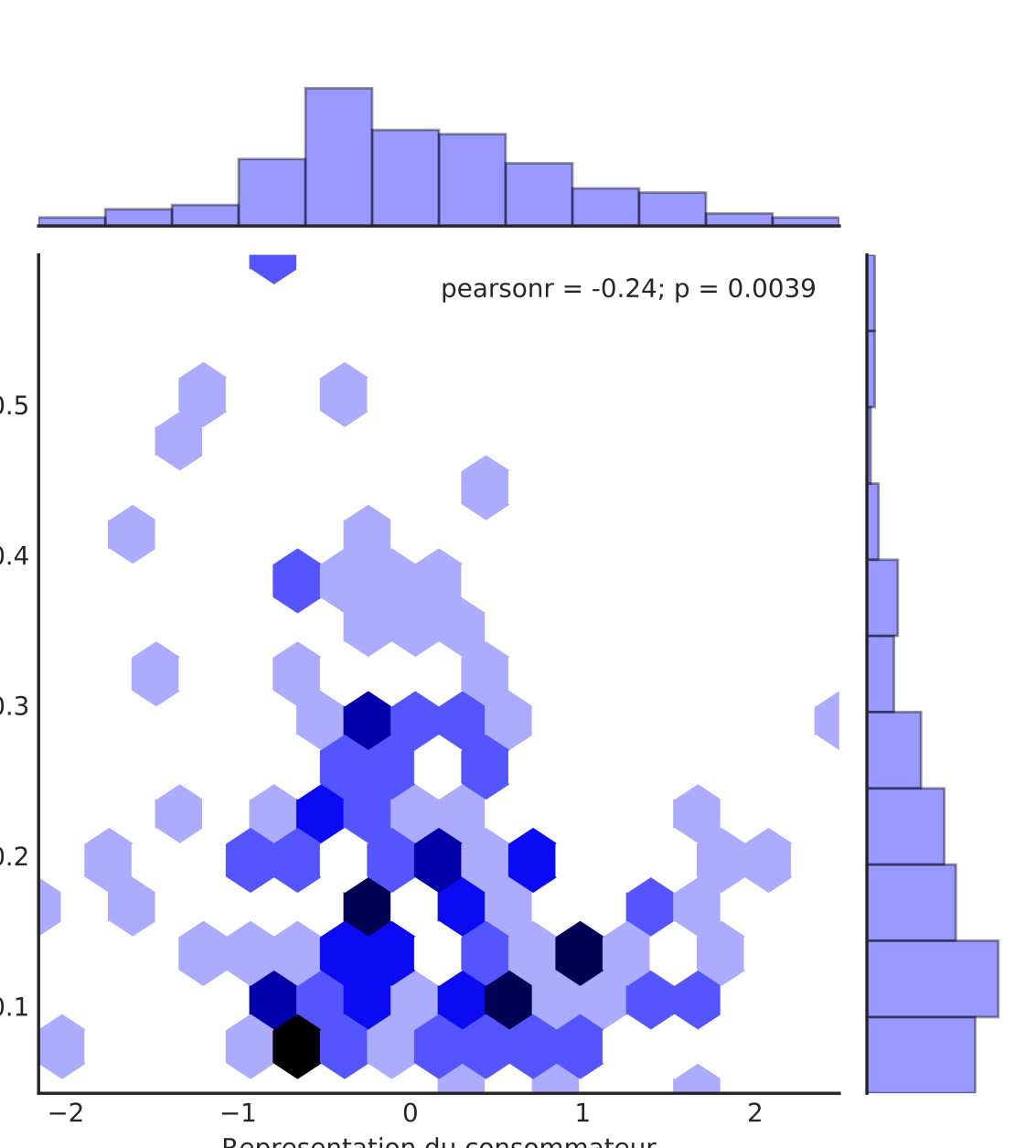

Figure 4: Distribution of $F_{1}$ measures relative to consumers (a) and products (b)

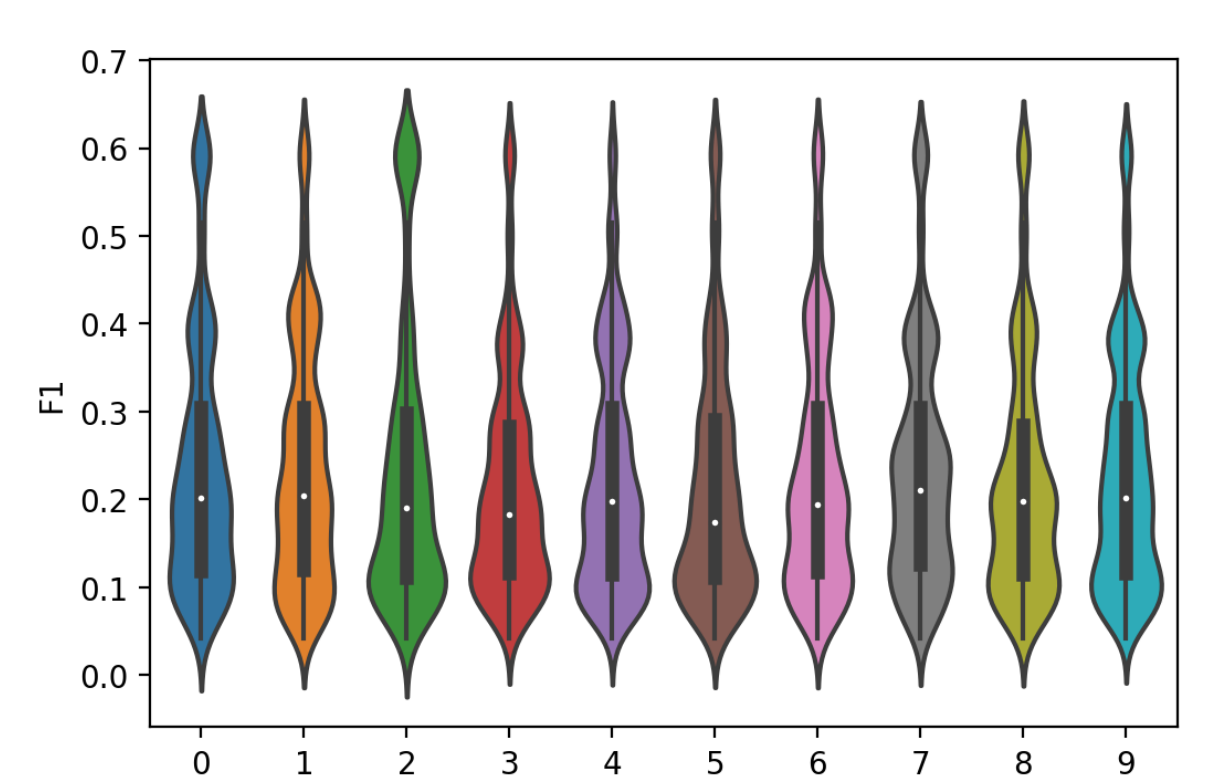

(a) (a)

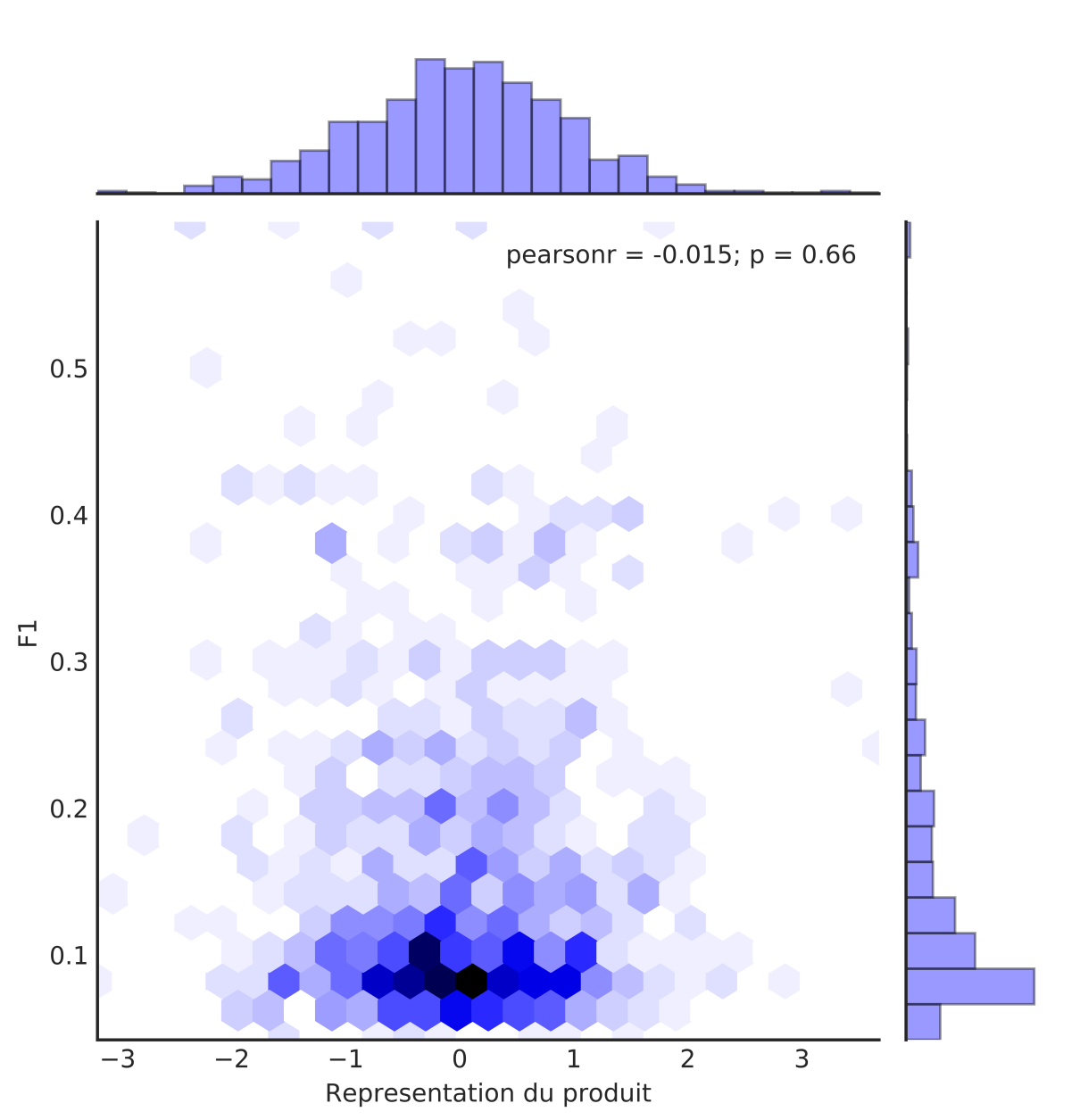
consumers

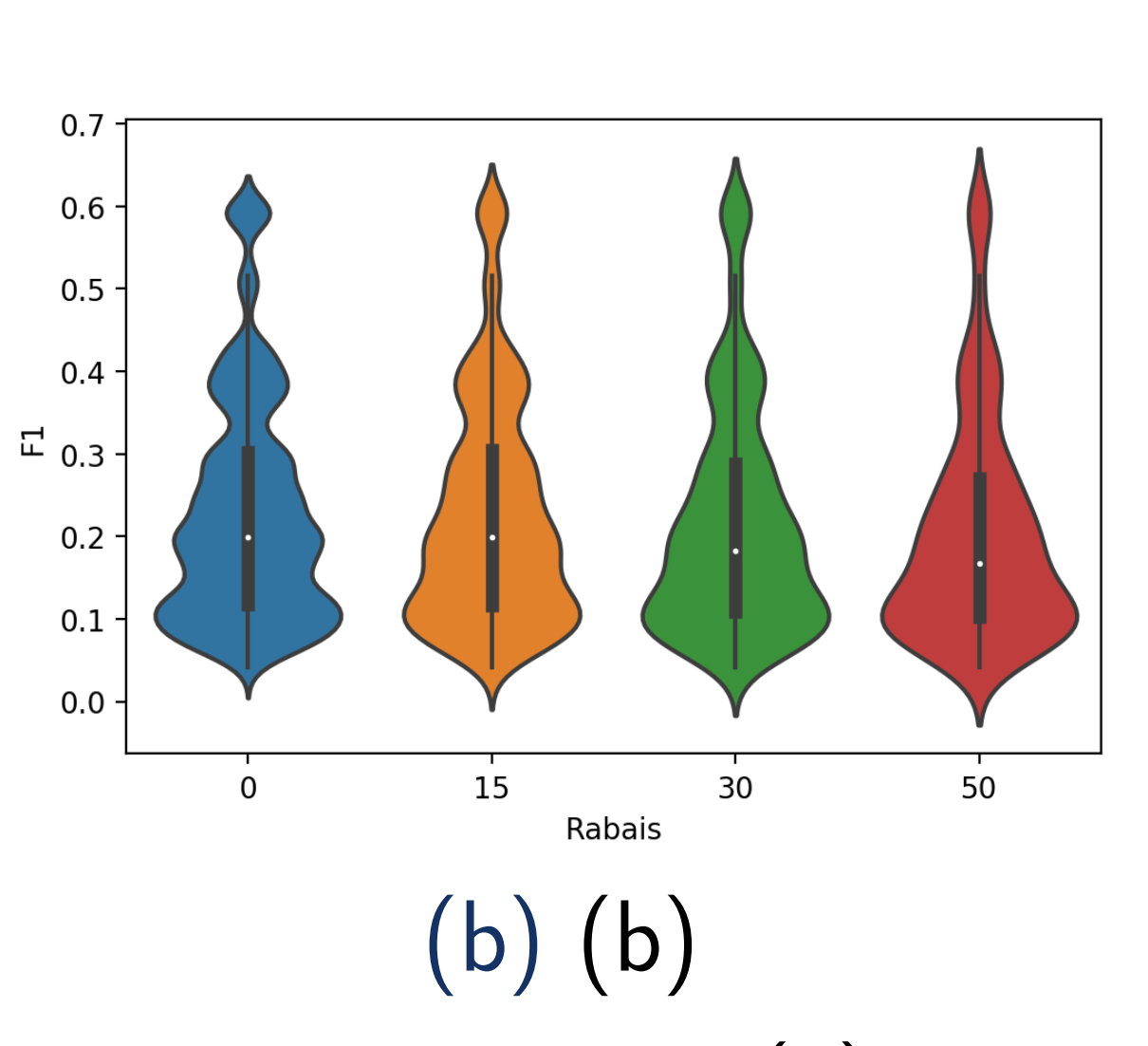
Figure 5: Distribution of $F_{1}$ measures against stores (a) and rebates (b).
Conclusion

- We modeled the habits of consumers on CircuitPromo.ca by using deep neural networks. - We used two types of neural networks during learning: recurrent neural networks (RNN) and Feedforward neural networks.

- The value of the $F_{1}$ statistic that represents the quality of our model is 0.22 . The constant influx of new data on CircuitPromo will improve the model over time.

- The originality of our approach, compared to existing algorithms, is that in addition to the purchase history we also consider promotions, possible purchases in different stores and the distance between these stores and the consumer's home.

\section{References}

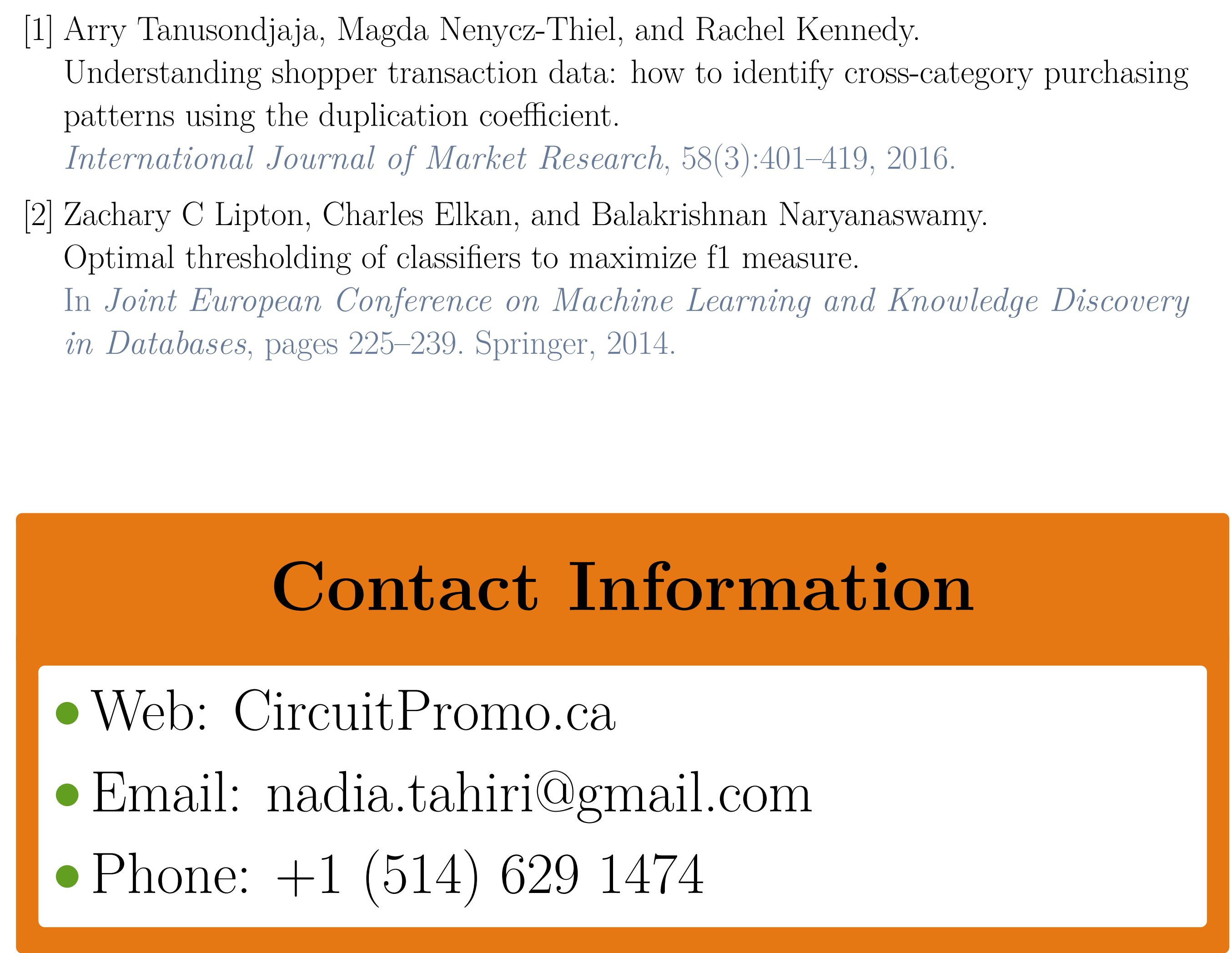

\section{UQĀM}

